\title{
O PROCESSAMENTO DE NARRATIVAS EM SEGUNDA LÍNGUA: UM ESTUDO COM ANIRS
}

\author{
Lilian Cristine Scherer ${ }^{1}$ \\ Gislaine Machado Jerônimo ${ }^{2}$ \\ Ana Inés Ansaldo ${ }^{3}$
}

\begin{abstract}
This article presents the results of a study which investigated the performance of native speakers of English (L1) when reading texts in French (L2), language in which they had intermediate proficiency. The study investigated, in relation to behavioral performance, these readers' accuracy and response times when processing the text at three different levels (the macro- and the micro-structure and the situational model), whereas, with respect to brain activity, the study analyzed the participation of the two cerebral hemispheres in processing the three levels mentioned above, using the fNIRS (functional Near-Infrared Spectroscopy) neuroimaging tool. The behavioral results show statistically significant differences between the two groups. The neuroimaging results indicate a statistically significant participation of the two regions of interest in temporal areas of the right hemisphere during processing at the macro-structure, as well as of temporal and frontal regions in the left hemisphere in comprehension at the microstructure.
\end{abstract}

Keywords: Neuropsicolinguistics, Second Language Reading, fNIRS, Text Processing.

\footnotetext{
1 Faculdade de Letras - Linguística, Pontifícia Universidade Católica do Rio Grande do Sul PUCRS. LCS agradece à CAPES pela concessão de bolsa de Estágio de Doutorado a qual possibilitou a execução da pesquisa aqui apresentada.

2 Faculdade de Letras - Linguística, Pontifícia Universidade Católica do Rio Grande do Sul - PUCRS

3 Université de Montréal (UdeM) e Institut Universitaire de Gériatrie de Montréal (CRIUGM) - Canadá 


\section{INTRODUÇÃO}

O estudo do bilinguismo é relevante para que se obtenha uma compreensão maior sobre como as línguas e seus componentes são representados, armazenados e computados no cérebro. Como aponta Vaid (2002), esse tipo de estudo nos possibilita compreender melhor a relação cérebro e linguagem. Mais especificamente, consegue-se identificar como os hemisférios cerebrais participam no processamento das línguas como um todo, bem como de cada um de seus componentes - os aspectos sintáticos, semânticos, pragmáticos, fonológicos, entre outros (BEEMAN; CHIARELLO, 1998). Dentre esses componentes, o processametno de texto tem sido um dos menos estudados, especialmente com o uso de técnicas de neuroimagem, devido às restrições impostas pelas técnicas, uma vez que a maioria delas dificulta a apresentação de tarefas mais extensas que o nível da frase, bem como a interpretação dos dados.

Neste artigo, apresentaremos dados obtidos por meio da implementação de um estudo comportamental e de neuroimagem, desenvolvido com o intuito de verificar como falantes com proficiência intermediária na segunda língua (L2) ${ }^{4}$ compreendem textos nessa língua. Inicialmente, serão abordadas questões referentes à organização e ao processamento das línguas no cérebro bilíngue. Logo depois, traremos as definições de micro, macroestrutura e modelo situacional referentes à compreensão do discurso ${ }^{5}$ narrativo. Em seguida, serão apresentados alguns dados comportamentais e de neuroimagem sobre o tratamento do discurso na L2. Finalmente, será exposto o estudo desenvolvido, seguido da discussão dos seus resultados.

\section{REPRESENTAÇÃO E PROCESSAMENTO DA L2 NO CÉREBRO BILIINGUE}

Os pesquisadores não são unânimes ao definir o que venha a ser um bilíngue. A concepção a ser usada neste trabalho é a proposta por

4 Neste artigo, os termos "segunda língua" (L2) e "língua estrangeira" (LE) serão empregados indistintamente. Da mesma forma, não será feita aqui distinção entre os termos "aquisição" e "aprendizagem" de segunda língua.

5 Não é propósito do presente trabalho problematizar os conceitos "texto" e "discurso". Para não causar problemas de compreensão e, por vezes, discussões teóricas improcedentes, já que "alguns chamam de texto o que outros chamam de discurso e vice-versa" (Travaglia, 1991), utilizaremos aqui os termos "texto" e "discurso" como sinônimos. 
Vaid (2002), segundo o qual bilíngue é um indivíduo que possui conhecimento de e regularmente usa duas línguas, embora as duas línguas não precisem necessariamente ser usadas no mesmo contexto ou conhecidas no mesmo nível. Dificilmente um bilíngue consegue atingir um nível de proficiência na L2 (e nas demais línguas, no caso dos multilíngues) do mesmo modo como atinge na sua língua materna (L1) (GROSJEAN, 1998). A língua é composta por vários componentes (sintaxe, semântica, discurso, entre outros) e engloba a compreensão e uso de quatro habilidades (leitura, fala, escrita e compreensão auditiva). Portanto, adquirir uma alta proficiência em cada um dos componentes e das habilidades é uma tarefa bastante complexa. A heterogeneidade na proficiência atingida nesses níveis decorre de vários fatores, dentre os quais citam-se a maneira e a idade com que adquirimos a L2, o contexto e a frequência de uso efetivo de cada um dos componentes e habilidades, a quantidade de exposição a ensino formal ministrado na língua alvo, bem como a distância estrutural entre a L1 e a L2.

Com relação à idade, pesquisadores discutem a respeito da existência ou não de um período crítico que seria favorável à aquisição de uma L2. Friederici e Wartenburger (2010) defendem a importância de aprender uma L2 nos anos iniciais de vida, visto que os resultados em termos de proficiência seriam melhores. Posição essa que Bialystok (2010) critica, pois a autora acredita ser o uso um fator determinante na proficiência e não o período de aquisição.

No intuito de compreender essas diferenças no processamento da L2 entre bilíngues, muitos aspectos correlacionados têm sido o escopo de pesquisas recentes. Dentre elas, destacam-se: 1 . os estudos sobre a representação cortical da L1 e da L2 (se o substrato cortical para a linguagem é o mesmo em monolíngues e bilíngues, se ou quando o processamento da L2 recruta as mesmas regiões envolvidas no processamento da L1, bem como a influência da tipologia de tarefas na ativação cerebral) (ABUTALEBI; GREEN, 2007; GÓMEZ-RUIZ, 2010), 2. o papel da idade de aquisição da L2 (FRIEDERICI; WARTERNBURGER, 2010) e da forma de aquisição (se em contexto natural ou formal, como numa sala de aula), 3. a influência do nível de proficiência na organização e armazenamento da L2 (FABRO, 2001; BIALYSTOK, 2006) e 4 . o impacto da frequência de uso de ambas as línguas em tarefas cotidianas (ZIMMER, 2010) 
Evidências aportadas por esses e outros estudos têm mostrado que dois dos mais importantes fatores a determinar o padrão comportamental e cerebral de processamento da L2 são a sua frequência de uso e a proficiência alcançada na referida língua. Essa conclusão aponta para a necessidade de observarem-se esses dados na análise dos resultados obtidos nos experimentos.

Uma questão importante que subjaz aos estudos sobre a estruturação das línguas no cérebro é a maneira como o seu conhecimento é armazenado no sistema de memória. Os indivíduos precisam combinar dois tipos de memórias: a procedural e a declarativa. A memória procedural relaciona-se com o uso automático e não-consciente de características governadas por regras como as sintáticas, fonológicas, morfológicas e lexicais, enquanto a memória declarativa engloba propriedades como o significado de palavras e seus sons. Os dois sistemas são independentes, mas interconectados. Da mesma forma, não se pode postular que, por causa da existência desses dois sistemas, duas línguas sejam localizadas em regiões distintas. Dados advindos de estudos com técnicas de neuroimagem e com estimulação cortical têm confirmado a existência de redes sobrepostas entre as línguas, com uma diferenciação maior quanto às áreas ativadas no caso das línguas menos proficientes (PARADIS, 2004). Em outras palavras, segundo o autor, as duas línguas no cérebro de um bilíngue são neurofuncionalmente independentes, "embora sua representação não seja anatomicamente independente [...], embora cada sistema linguístico possa ser articulado por circuitos neuronais diferentes" (PARADIS, 2004, p. 190).

Paradis acrescenta, ainda, que o que difere entre monolíngues e bilíngues é o que é processado (ou seja, as estruturas internas dos conteúdos), mas não como a informação é processada (ou seja, os princípios pelos quais as línguas são representadas e processadas).

Ligada também à discussão sobre o armazenamento das línguas no cérebro, está a questão de como são acessadas as línguas nos sistemas de memória. Segundo Green (1998) e Green e Price (2001), quanto mais uma palavra, expressão ou língua é usada e quanto mais recente tiver sido seu uso em relação à sua tradução equivalente, mais baixo será seu limiar de ativação, ou seja, mais facilmente ela será ativada. Se, ao contrário, a informação não é utilizada com frequência ou não foi utilizada recentemente, o seu limiar de ativação será alto, o que dificultará sua 
evocação num contexto bilíngue. Portanto, a disponibilidade de uma palavra, expressão ou língua está diretamente relacionada à frequência e à recenticidade de seu uso.

Ainda considerando-se a questão do uso da língua, Ullman (2010) propõe o modelo declarativo/procedural de linguagem - DP Model. Esse modelo alia o uso à memória. Ullman prevê a possibilidade de o conhecimento explícito se tornar implícito a partir do uso, da exposição e da prática com o idioma. Isso quer dizer que quanto mais o indivíduo for exposto e praticar a L2, mais chances ele terá de transformar seu conhecimento declarativo em procedural e solidificar determinada aprendizagem.

\section{O PROCESSAMENTO DO TEXTO NARRATIVO}

Narrativas são utilizadas extensivamente para a comunicação humana; tanto a compreensão como a produção oral e escrita de narrações constitui parte fundamental da nossa experiência. Desse modo, nossa experiência é altamente influenciada pelo ato de compreender e produzir narrativas (MAR, 2004). Além disso, é um dos primeiros gêneros discursivos de que a criança lança mão. $\mathrm{O}$ que intriga estudiosos tanto da Psicologia Cognitiva como da Linguística e Neurociência são os processos que subjazem a essa habilidade linguística.

Segundo Mar (2004), em um levantamento a respeito da neuropsicologia da narrativa, os estudos que abordam o processamento da narrativa dividem-se entre os que priorizam a compreensão e os que tratam da produção do discurso, ambas podendo ser tanto orais quanto escritas. Ambos os processos são habilidades linguísticas e cognitivas de grande importância comunicativa. Dentre eles, a produção é o aspecto mais negligenciado no processamento da linguagem por psicolinguistas. Ressaltam-se os estudos que abordam a compreensão tanto auditiva (em maior número) como leitora. Mar (2004) salienta que, embora teorias iniciais a respeito da compreensão do discurso tivessem foco especial na representação do texto pelo leitor, a maior parte dos modelos modernos concorda com a relevância de se entender como o leitor representa o que é dito no texto e cita como sendo um dos modelos mais estudados o de Van Dijk e Kintsch (1983), modelo que utilizaremos para a análise dos nossos dados. 
No que tange à compreensão do discurso, esta passa por complexos níveis de representação: a micro-estrutura, a macro-estrutura e o modelo situacional. Tal distinção de elementos do texto foi proposta pelo linguista Van Dijk e pelo psicólogo Kintsch, por meio de uma parceria entre a Linguística e a Psicologia Cognitiva (Kintsch; Van Dijk, 1978). Essa proposta de três níveis de compreensão do discurso surge pela necessidade de se estudar a forma como os indivíduos compreendem o discurso como um todo, uma vez que os estudos anteriores se detinham apenas na compreensão da palavra ou da frase (Van Dijk, 2010).

Os estudos de Van Dijk sobre a linguagem visam atingir a forma como o texto relaciona diferentes sentenças para transmitir significado. Sua preocupação é com a noção de coerência (Van Dijk, 1993). Primeiramente, essa noção se ocupa apenas da relação entre uma frase com a frase seguinte. Depois surge uma noção essencial para o significado do texto: a macroestrutura, vista como uma estrutura cognitiva de significado mais geral que traz unidade e coerência ao texto. A macro-estrutura pode ser compreendida como uma forma de resumo de uma história.

A estrutura de superfície, isto é, as palavras e sintagmas do texto, seria a micro-estrutura - o conteúdo semântico local do texto, que se transforma em macro-estrutura, em um conteúdo mais global, a partir de "regras de mapeamento", como cancelamento de detalhes não importantes, generalização de situações, construções de novas sequências frasais, entre outras. Já o modelo situacional representa a situação que é descrita no texto, retirada da estrutura do texto e encaixada ao conhecimento de mundo do leitor (VAN DIJK; KINTSCH, 1983).

Desse modo, a compreensão de um texto ou discurso implica a criação de um texto base, que organiza hierarquicamente todas as proposições explícitas no texto, estabelecendo relações de significado ao nível da frase (micro-estrutura) e alcançando uma estrutura mais geral de significado, a macro-estrutura. Assim, a compreensão do texto combina conhecimento sobre a linguagem e conhecimento sobre o mundo (KINTSCH, 1988).

Contudo, para que o indivíduo alcance os três níveis de compreensão do discurso, é necessária uma participação efetiva de ambos os hemisférios cerebrais e não apenas do hemisfério esquerdo (HE), como apontava a literatura clássica sobre o assunto (SPRINGER; DEUTSCH, 1998). A partir da década de 1950, pesquisas mostraram que o HE ten- 
de a estar envolvido na coerência mais local (na micro-estrutura), enquanto que áreas do hemisfério direito (HD) estariam mais implicadas na coerência global (na macro-estrutura da mensagem) (ST GEORGE et al., 1999; ROBERTSON et al., 2000; GERNBACHER; KASCHAK, 2003). Tal distinção processual pode significar uma complementaridade hemisférica (BEEMAN; BOWDEN; GERNSBACHER, 2000) na execução de algumas funções da linguagem e não somente a dominância de um lado do cérebro em relação ao outro.

\section{A PESQUISA SOBRE O PROCESSAMENTO DO DISCURSO POR BILIINGUES}

\subsection{Evidências advindas de estudos comportamentais}

Alguns dos temas mais investigados por estudos comportamentais sobre o tratamento do discurso por bilíngues são o papel da proficiência na compreensão de textos e a questão do uso de estratégias de leitura e de sua transferência ou não da L1. Há ainda alguns estudos que tratam do impacto da leitura em L2 sobre a leitura em L1.

Para ilustrar o primeiro tema na agenda de pesquisa, pode-se citar o estudo desenvolvido por Chen e Donim (1997), que investigou o papel da proficiência linguística na L2 e do conhecimento específico do assunto tratado no texto no processamento do discurso por bilíngues. Os pesquisadores verificaram que o conhecimento do conteúdo era mais relevante para a compreensão dos níveis mais elevados do discurso do que para a computação de aspectos mais locais, sintáticos e semânticos. Já a proficiência na L2 consistentemente afetou o processamento de ordem mais inferior (mais centradas nos aspectos formais, gramaticais, do texto), mas não foi decisiva na compreensão de ordens mais elevadas no discurso.

Outro estudo, conduzido por Walter (2004), investigou a transferência de estratégias de leitura da L1 (francês) para a L2 (inglês) por falantes de proficiência elevada (intermediária avançada) e menos elevada (intermediária). Seu estudo revelou que alunos mais proficientes conseguem aplicar com mais sucesso estratégias de leitura para lidar com o texto escrito na $\mathrm{L} 2$, ao passo que os menos proficientes não conseguem, mesmo quando eles não sentem problemas em processar as sentenças dos textos. A autora concluiu que os falantes proficientes obtêm êxito ao transferir para a L2 a habilidade de construir corretamente representações mentais 
dos textos e que essa habilidade está ligada ao desenvolvimento da memória de trabalho aplicada ao processamento da L2.

Pesquisadores belgas fizeram um estudo bastante inovador e curioso. Considerando que o fato de que tornar-se bilíngue traria algumas vantagens cognitivas, Van Assche e colegas (2009) se propuseram a avaliar o impacto que essa habilidade causaria na língua materna. Mais especificamente, queriam investigar como o conhecimento de uma L2 influenciaria a forma como as pessoas leem na L1. Utilizaram a técnica de monitoramento ocular de palavras cognatas em textos e contaram com a participação de 42 sujeitos bilíngues tardios. Ao final do estudo, demonstraram que a acurácia na habilidade leitora em L1 sofreu alterações positivas devido ao fato de os sujeitos possuírem conhecimento de uma L2.

\subsection{Evidências advindas de estudos com neuroimagem}

Estudos desenvolvidos com neuroimagem têm possibilitado a comparação e o refinamento de dados advindos de estudos comportamentais. Um aspecto confirmado na maioria dos trabalhos é o de que existe uma maior variabilidade nos padrões de ativação cerebral no processamento da L2 por bilíngues menos proficientes. Essa variabilidade é refletida, por exemplo, na ativação adicional de áreas mesio-temporais, parahipocampais e pré-frontais, conhecidas por estarem envolvidas, respectivamente, na memória declarativa e no controle consciente, e em áreas do hemisfério direito, envolvido, entre outras atividades, com a inferenciação pragmática (PARADIS, 2004). Desse modo, ocorre uma maior dispersão e uma menor ativação nas áreas perisilvianas tradicionais. Uma explicação plausível para a variabilidade seria que a variação no processamento da L2 por falantes não-proficientes, em oposição à maior homogeneidade verificada nos padrões de ativação da L1, automatizada, poderia retratar uma aplicação de estratégias compensatórias recrutadas para lidar com uma reduzida competência implícita.

As técnicas de neuroimagem mais utilizadas para o estudo do processamento do discurso têm sido a imagem por ressonância magnética funcional (IRMf ou fMRI, sigla em inglês) e a tomografia por emissão de pósitron (TEP ou PET, em inglês). Estudos de neuroimagem com textos apresentados visualmente são raros, provavelmente como uma consequência das limitações impostas pelas técnicas para esse tipo de 
modalidade de apresentação. Um exemplo de estudo sobre a leitura de texto por bilíngues é o desenvolvido por Kobayashi e colegas (2006), que analisaram a compreensão da teoria da mente por bilíngues falantes de japonês e inglês e por monolíngues falantes de inglês. Os autores pretendiam examinar a influência da cultura e da língua nas bases neuronais relacionadas ao processamento da teoria da mente, uma vez que resultados incongruentes haviam sido reportados na literatura sobre a compreensão desse aspecto por populações com diferentes culturas. Nesse experimento, ambos os grupos de cultura/língua recrutaram vários correlatos neuronais, incluindo o córtex medial pré-frontal e o córtex cingulado anterior; no entanto, algumas outras áreas tais como o giro frontal inferior foram empregados de uma forma específica segundo a língua/cultura, sugerindo que o modo como os adultos compreendem a teoria da mente não é completamente universal.

Leonard et al (2011), em um estudo que trata do impacto do nível de proficiência na L2 sobre as áreas cerebrais recrutadas para a compreensão de palavras ouvidas e lidas, corroboram os achados da literatura. Os pesquisadores combinaram o uso de fMRI com o da magnetoencefalografia (MEG). Em seus resultados perceberam que a falta de proficiência na L2 determinou o recrutamento de áreas cerebrais não-clássicas para o processamento das palavras. Nos bilíngues menos proficientes houve maior ativação nas regiões visuais superiores tanto para a modalidade visual como auditiva.

Uma análise de dados aportados por estudos sobre a compreensão de texto veiculado oralmente (PERANI et al., 1998 e 1996; DEHAENE et al., 1997) e a produção de discurso oral (WATTERNDORF et al., 2001; KIM et al., 1997) reflete, de um modo geral, uma ativação bilateral e mais difusa, especialmente no hemisfério direito, quando o participante da pesquisa apresenta níveis mais baixos de proficiência e em aquisição mais tardia da L2 (depois da infância ${ }^{6}$ ). Com relação ao processamento da L1, áreas perisilvianas revelaram maior ativação. Outro dado importante diz respeito à possível diferenciação no funcionamento da área de Broca. Algumas pesquisas sugerem uma especiali-

6 Estudos têm trazido dados nem sempre congruentes no sentido de apontar uma relação entre a idade de aquisição e a organização da L2 no cérebro. Tem havido uma tendência a se enfatizar o papel do uso efetivo da L2 e de sua recenticidade (aliados à proficiência) em detrimento do fator idade de aquisição para explicar a ativação da L2 de forma mais ou menos similar à da registrada na $\mathrm{L} 1$.

Organon, Porto Alegre, nº 51, julho-dezembro, 2011, p. 291-308 
zação diferenciada na área de Broca (mais especificamente na BA44 e BA45), a qual pode ser relacionada à idade e à forma de aquisição, à natureza da linguagem, ou ainda, segundo postulado por alguns estudos, à tipologia da tarefa ou dos domínios específicos da linguagem, como observado em um estudo desenvolvido por Newman e colegas (2003), o qual demonstrou diferentes padrões de ativação dentro da área de Broca para diferentes condições sintáticas e semânticas na L1.

Ainda sobre o processamento da narrativa em bilíngues salientamos outro estudo com neuroimagem que aborda a Língua de Sinais Americana (ASL). Newman et al (2010) estudaram não só o processamento da narrativa, mas também da prosódia. Esse estudo com bilíngues, que diferem na modalidade, sugere similar organização hemisférica. Segundo o estudo, o HE ocuparia uma função dominante com relação à organização da linguagem tanto falada como de sinais. Contudo, o estudo menciona a importância do HD em diferentes tipos e níveis de processamento da narrativa, incluindo a prosódia, expressão facial e estrutura do discurso narrativo. Foi utilizada a técnica de fMRI para contrastar o processamento de frases na ASL que continham informações narrativas com frases sem pistas narrativas. Os participantes tiveram robusta ativação na área perisilviana do córtex, bem como no gânglio basal, regiões mediais frontais e temporais. O HD teve maior ativação no giro inferior frontal e sulco temporal superior nas frases com informações narrativas, envolvendo áreas semelhantes às do processamento da narrativa na linguagem falada. Os resultados reportados nesse estudo fornecem informações que dão suporte à idéia de que todas as línguas naturais processam parte da linguagem em regiões do HE e confirmam a suposição de que o discurso narrativo em sua coerência global é associado a áreas do HD, o que permite inferir que tanto a língua de sinais como a oral são processadas similarmente.

A seguir, apresenta-se o experimento desenvolvido para investigar o processamento de texto narrativo por bilíngues, com o emprego da técnica fNIRS.

\section{O EXPERIMENTO}

O experimento aqui reportado foi desenvolvido utilizando uma técnica de neuroimagem não invasiva, com alta resolução temporal 
0 processamento de narrativas em segunda língua:...

e razoável resolução espacial, denominada fNIRS (functional NearInfrared Spectroscopy), no Centre de Recherche Institut Universitaire de Gériatrie de Montréal (CRIUGM), centro de pesquisa filiado à Universidade de Montreal, no Canadá. A técnica consiste na emissão de raios infravermelhos acoplados às regiões cerebrais a serem investigadas, juntamente com detectores, os quais captarão as mudanças hemodinâmicas decorrentes das ativações corticais resultantes da resolução das tarefas experimentais propostas. A colocação dos eletrodos (de emissão dos raios e de captação) obedece ao sistema de colocação utilizado pelo método empregado em Potenciais Relacionados a Eventos (PRE ou ERP, em inglês). A técnica tem como vantagem, em relação às outras, o fato de permitir o registro de imagens de regiões corticais de maneira ecologicamente mais válida, uma vez que o participante responde às tarefas acomodado confortavelmente diante do computador, sem interferência de ruídos gerados pelo equipamento. Movimentos devem ser controlados, porém o participante não fica tão imobilizado quanto deve permanecer ao se submeter à maioria das outras técnicas. Além disso, fNIRS tem um custo menos elevado que o uso de IRMf ou TEP.

O objetivo da pesquisa foi verificar a compreensão leitora de falantes nativos de inglês, aprendizes de francês, nos três diferentes níveis de compreensão do discurso - micro-estrutura (MIC), macro-estrutura (MAC) e modelo situacional (SIT) (KINTSCH, 1998). Dados comportamentais sobre acurácia e tempo de reposta foram coletados através da utilização do software E-Prime (http://www.pstnet.com), concomitantemente com a coleta de dados de neuroimagem.

Participou do estudo um grupo formado por 10 adultos jovens (doravante denominado JI) (6 do sexo masculino), bilíngues ( $\mathrm{L} 1=$ inglês, L2 = francês, em nível intermediário de proficiência), idade entre 21 e 34 anos (média 24.2), cuja escolaridade varia entre 13 e 20 anos (média 16.6). A idade de aquisição do francês varia entre 9 e 16 anos (média 11.2). Todos os participantes receberam instrução formal em francês, ou seja, frequentaram cursos de formação geral ministrados em francês de 4 a 12 anos (média 6.6). Para atestar sua proficiência na L2, os participantes responderam a um questionário que, dentre outros dados, solicitou uma auto-avaliação do domínio do francês nas quatro habilidades linguísticas, além de resolverem 
um teste standard, o DELF B, no qual obtiveram um escore entre 60 e 88 pontos (média 74.8). Ambos os instrumentos atestaram a proficiência intermediária dos participantes. Como grupo controle, participaram 10 adultos jovens falantes nativos de francês (doravante denominado grupo JF), 6 do sexo masculino, com idade entre 21 e 36 anos (média 25.7) e escolaridade entre 15 e 22 anos de estudos formais (média de 17.3).

Aplicaram-se testes neuropsicológicos para verificação das condições de capacidade de controle inibitório, atenção e memória de trabalho, a fim de possibilitar o cruzamento desses dados com o desempenho dos participantes, dados esses a serem explorados em artigo em preparação.

Para a verificação da compreensão leitora, os participantes leram 36 pequenas narrativas em francês, agrupadas em blocos nas 3 condições investigadas (MIC, MAC e SIT), seguidas de afirmações sobre cada narrativa. Essas afirmações deveriam ser julgadas como sendo verdadeiras ou falsas em relação ao conteúdo da narrativa lida. Os participantes deveriam pressionar a tecla indicando V (para verdadeiro) ou F (para falso) no teclado do computador. Uma sessão de treinamento antecedeu as aquisições. (Para maiores detalhes sobre o estudo, ver SCHERER, 2007). Quatro regiões de interesse (ROIs) foram selecionadas, bilateralmente: 2 regiões frontais - a inferior e uma mais mediana, abrangendo a área de Broca - e 2 regiões temporais, incluindo a área de Wernicke.

\section{APRESENTAÇÃO E DISCUSSÃO DOS RESULTADOS}

Os dados comportamentais, tratados pela aplicação dos testes estatísticos Kruskal-Wallis e Friedman, demonstraram diferenças significativas entre os dois grupos em termos da acurácia no processamento dos níveis mais elevados do discurso (MAC e SIT). O grupo JI (não proficiente em francês) revelou escores mais baixos também no processamento de MIC, porém não houve relevância estatística na comparação entre os grupos. Em termos de tempo de resposta, diferenças significativas ocorreram no processamento dos três níveis de compreensão das narrativas na comparação entre os grupos, uma vez que o grupo JI demonstrou um tempo de resposta significativamente superior ao do grupo JF. 
Os dados obtidos através da neuroimagem revelaram uma ativação significativa (ou seja, aumento dos níveis de HbO - oxi-hemoglobina) nas 4 regiões de interesse (ROIs) no hemisfério esquerdo durante o processamento de MIC pelo grupo JI, e um aumento significativo de HbR (desoxigenação) na região que abrange o lobo temporal superior esquerdo no grupo JF. As ativações não alcançaram índices significativos no processamento de SIT em ambos os grupos, ao passo que MAC gerou uma aumento significativo de $\mathrm{HbR}$ nas duas regiões temporais investigadas, no hemisfério direito, no grupo JI (ver Figura 1).

Uma análise geral dos padrões de ativação cerebral dos leitores de francês como L2 revela uma participação mais significativa de áreas cerebrais nesse grupo, bem como uma maior difusão nas ativações. Esses dados corroboram resultados anteriores, apresentados na literatura, os quais demonstraram que, ao produzirem ou compreenderem uma língua menos proficiente, os participantes recrutam áreas adicionais para lidarem com uma tarefa linguística.

Da mesma forma, comparando-se o papel dos dois hemisférios cerebrais, confirmam-se dados já reportados (FEDERMEIER; KUTAS, 1999). Mais especificamente, ressaltou-se aqui o papel do HE na compreensão mais local do texto em termos sintáticos e semânticos (aqui, na micro-estrutura textual), na comparação com o HD, essencial para processos de integração semântica no nível do discurso (aqui, representado pela macro-estrutura textual). Em outras palavras, enquanto o HE focaliza a coerência local, entre e dentro de frases, o HD se encarrega da coerência global, na macro-estrutura da mensagem.

Comparando-se dados de neuroimagem com os comportamentais, pode-se pressupor que a ativação significativa no processamento de MIC pelo grupo JI (falantes não nativos de francês) no HE demonstrou uma acertada estratégia de processamento, uma vez que o resultado comportamental demonstrou altos níveis de acurácia. Por outro lado, uma ativação de regiões temporais no processamento da MAC por esse grupo levou a baixos níveis de acurácia, revelando uma possível escolha errônea de estratégias para lidar com a macro-estrutura do texto, uma vez que as regiões frontais do HD são mais relacionadas ao processamento desse nível textual (JOANETTE; BROWNELL, 1990).

Organon, Porto Alegre, nº 51, julho-dezembro, 2011, p. 291-308 


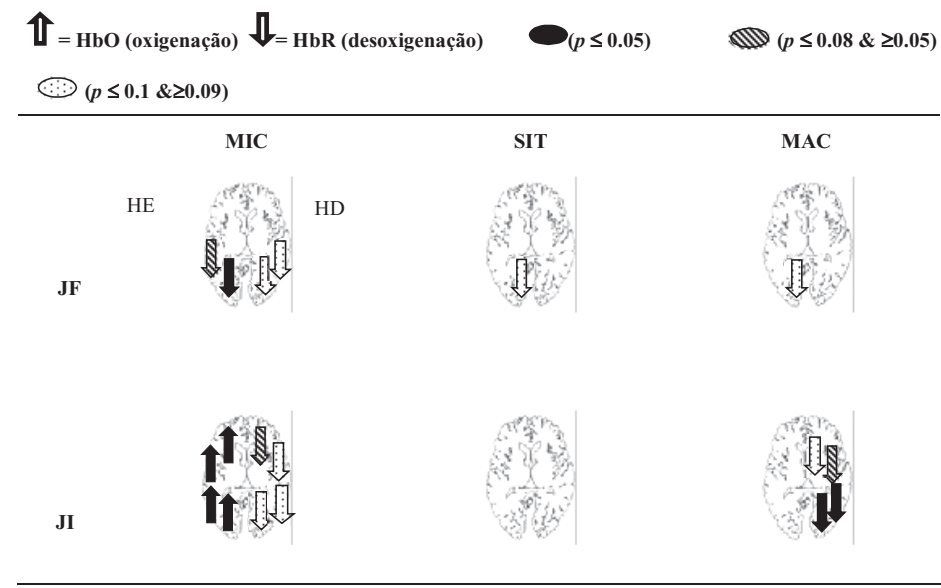

Fig 1: Representação dos padrões hemodinâmicos nas ROIs por grupo e condição

Finalmente, os dados parecem trazer evidências para confirmar a ocorrência de uma cooperação entre os hemisférios cerebrais para lidar com alguma questão complicadora (MAERTENS, POLLMANN, 2005), no caso, o processamento linguístico, visualizado em especial no processamento da micro-estrutura.

\section{CONSIDERAÇÕES FINAIS}

A organização das línguas no cérebro bilíngue ou multilíngue é uma fascinante arena de investigação, ainda muito incipiente. As técnicas de neuroimagem surgem como uma importante ferramenta para ampliar nosso conhecimento sobre os construtos neuropsicolinguísticos associados ao processamento das línguas, relacionando-se esse processamento ao papel dos hemisférios cerebrais e de componentes cognitivos diretamente envolvidos, como os diferentes tipos de memórias e a capacidade de atenção e de inibição. No entanto, cabe ressaltar a relevância da associação desses estudos com dados empíricos comportamentais, os quais fornecem importantes fontes de reflexão no momento em que se interpretam esses dados à luz de evidências aportadas pela neuroimagem.

Desse modo, poderá ocorrer um avanço no sentido de se proporem teorias que procurem explicar o processamento linguístico por bilín- 
gues. O surgimento dessas bases teóricas mais consistentes possibilitará também um aprimoramento no diagnóstico e no tratamento de deficiências linguísticas congênitas ou adquiridas.

\section{BIBLIOGRAFIA}

ABUTALEBI, J.; GREEN, D. W. Bilingual language production: The neurocognition of language representation and control. Journal of Neurolinguistics, 20, 242-275, 2007.

BEEMAN, M.; BOWDEN, E.; GERNSBACHER, M. Right and left hemisphere cooperation for drawing predictive and coherence inferences during normal story comprehension. Brain and Language, 71, 310-336, 2000.

BEEMAN, M.; CHIARELLO, C. Commentary: Getting the right meaning from discourse - Concluding remarks: getting the whole story right. In Mark Beeman and Christine Chiarello (Eds.) Right hemisphere language comprehension: Perspectives from cognitive science. New Jersey: LEA, 1998.

BIALYSTOK, E. The impact of bilingualism on language and literacy development. In T. Bhatia \& W. Ritchie (Eds.), The handbook of bilingualism, 577-601). New York: Blackwell, 2006.

BIALYSTOK, E; PETTITO, L.; GAZZELLONE, P. Language and Learning. Entrevista concedida ao programa de televisão: Your Voice, 2010. [online] Disponível em http://www.youtube.com/watch?v=hW_ qpta6zb4\&playnext=1\&list=PL17A5DB33BA6E0EA2. Arquivo acessado em 02 de março de 2011.

CHEN, Q; DONIN, J. Discourse processing in first and second language biology texts: effects of language proficiency and domain-specific knowledge. Modern Language Journal, 81, 2, 209-227, 1997. DEHAENE, S.; DUPOUX, E.; MEHLER, J.; COHEN, L.; PAULESU, E.; PERANI, D.; VAN DE MOORTEL, P.; LEHERICY, S.; LE BIHAN, D. Anatomical variability in the cortical representation of first and second language. Neuroreport, 8, 3809-3815, 1997.

FABRO, F. The Bilingual Brain: Cerebral Representation of Languages. Brain and Language, 79, 211-222, 2001. 
FEDERMEIER, K.D.; KUTAS, M. Right words and left words: electrophysiological evidence for hemispheric differences in meaning processing. Cognitive Brain Research, 8, 373-392, 1999.

FRIEDERICI, A.; WARTENBURGER, I. Language and brain. Wiley Interdisciplinary Reviews: Cognitive Science, 1(2), 150-159, 2010.

GERNSBACHER, M. A.; KASCHAK, M. P. Neuroimaging Studies of Language Production and Comprehension. Annual Review of Psychology. V.54, p. 91-114, 2003.

GOMEZ-RUIZ, M.I.. Bilinguism and the brain: myth and reality. Neurología, 25 (7): 443-452, 2010.

GREEN, D.W.; PRICE, C. Functional imaging in the study of recovery patterns in bilingual aphasia. Bilingualism: Language and Cognition, 4: 191-201, 2001.

GREEN, D.W. (1998). Mental control of the bilingual lexico-semantic system. Bilingualism. Language and Cognition, 1, 67-81.

GROSJEAN, F. Studying bilinguals: Methodological and conceptual issues. Bilingualism: Language and Cognition, 1: 131-149, CUP, 1998. KOBAYASHI, C; GLOVER, G.H.; TEMPLE, E. Cultural and linguistic influence on neural bases of 'theory of mind': An fMRI study with Japanese bilinguals. Brain and Language, 98, 210-220, 2006.

JOANETTE, Y., BROWNELL, H.H. Discourse Ability and Brain Damage: Theoretical an Empirical Perspectives. New York: Springer-Verlag, 1990.

KIM, K.H., RELKIN, N.R., LEE, K.M., HIRSCH, J. Distinct cortical areas associated with native and second languages. Nature, 388, 171174, 1997.

KINTSCH, W.; VAN DIJK, T.A. Toward a model of text comprehension and production. Psychological Review, 85, 363-394, 1978.

KINTSCH, W. The role of knowledge in discourse comprehension: A construction-integration model. Psychological Review, v. 95, n. 2, p. 163-182, 1988.

KINTSCH, W. Comprehension: a paradigm for cognition. New York: Cambridge University Press, 1998.

LEONARD, M. et al. Language proficiency modulates the recruitment of non-classical language areas in bilinguals. In Bilinguals. Plos ONE 6 (3): e18240. doi:10.1371/journal.pone.0018240, 2011.

MAERTENS, M.; POLLMANN, S. Interhemispheric resource sharing: Decreasing benefits with increasing processing efficiency. Brain and Cognition, 58, 183-192, 2005. 
MAR, R.A. The neuropsychology of narrative: story comprehension, story production, and their interrelation. Neuropsychologia 42, 14141434, 2004.

NEWMAN, S.D.; JUST, M.A.; KELLER, T.A.; ROTH, J.; CARPENTER, P.A. Differential effects of syntactic and semantic processing on the subregions of Broca's area. Cognitive Brain Research, 16, 297-307, 2003.

NEWMAN, A.J.; SUPALLA,T.; HAUSER, P.C.; NEWPORT, E.L. ;

BAVELIER, D. Prosodic and narrative processing in American Sign Language: an fMRI study. Neuroimage, 52, p. 669-676, 2010.

PARADIS, M. A Neurolinguistic Theory of Bilingualism (Studies on Bilingualism, 18).

Amsterdam: John Benjamins Publishing Co, 2004.

PERANI, D.; DEHAENE, S.; GRASSI, F.; COHEN, L.; CAPPA, S.F.; DUPOUX, E.; FAZIO, F.; MEHLER, J. Brain processing of native and foreign languages. NeuroReport, 7, 2439-2444, 1996.

PERANI, D.; PAULESU, E.; SEBASTIAN-GALLÉS, N.; DUPOUX, E.; DEHAENE, D.; BETTINAEDI, V.; CAPPA, S.; FAZIO, F.; MEHLER, J. The bilingual brain: Proficiency and age of acquisition of the second language. Brain, 121: 1841-1852, 1998.

ROBERTSON, D.A.; GERNSBACHER, M.A.; GUIDOTTI, S.J.;

ROBERTSON, R.R.W.; IRWIN, W.; MOCK, B.J.; CAMPANA, M.E. Functional neuroanatomy of the cognitive process of mapping during discourse comprehension. Psychol. Sci. 11, 255-260, 2000.

SCHERER, L.C. The impact of aging and language proficiency on the interhemispheric dynamics for discourse processing: a NIRS study. Tese de doutorado não publicada. UFSC, 2007.

SPRINGER, S.P; DEUTSCH G. Cérebro Esquerdo, cérebro direito. 2.ed. São Paulo: Summus, 1998.

St. GEORGE, M., KUTAS, M., MARTINEZ, A., SERENO, M.I. Semantic integration in reading: engagement of the right hemisphere during discourse processing. Brain 122, 1317-1325, 1999.

TRAVAGLIA, Luiz Carlos. Um estudo textual-discursivo do verbo no português do Brasil. Tese de doutorado, Campinas, 1991. [online] Disponível em http://www.mel.ileel.ufu.br/homepages/travaglia/artigos/ TESE_DOUTORADO_LUIZ_CARLOS_TRAVAGLIA.pdf. Arquivo acessado em 03 de março de 2011.

Organon, Porto Alegre, nº 51, julho-dezembro, 2011, p. 291-308 
ULLMAN, M.; SANZ, C.; GELFAND, M.; BOWDEN, H. Verbal Inflectional Morphology in L1 and L2 Spanish: A Frequency Effects Study Examining Storage Versus Composition. Language Learning 60:1, pp. 44-87, March 2010.

VAID, J. Bilingualism. Encyclopedia of the Human Brain, Vol. 1: 417434, Elsevier Science, USA, 2002.

VAN ASSCHE, E.; DUYCK, W.; HARTSUIKER, R.; DIEPENDAELE, $\mathrm{K}$. Does bilingualism change native-language reading? Psychological Science. Vol. 20, No. 8. p.923-927, 2009.

VAN DIJK, T. A.; KINTSCH, W. Strategies of discourse comprehension. New York: Academic Press, 1983.

VAN DIJK, T.A. Principles of critical discourse analysis. Discourse \& Society, 4, 249-283, 1993.

VAN DIJK, T. A. Cognição: discurso e interação. São Paulo: Contexto, 2010. WALTER, C. Transfer of reading comprehension skills to L2 is linked to mental representations of text and to L2 working memory. Applied Linguistics, 25, 3, 315-339, 2004.

WATTENDORF, E. ; WESTERMANN, B.; ZAPPATORE, D.; FRANCESCHINI, R.; LÜDI, G.; RADÜ, E-W.; NITSCH, C. Different language activate different subfields in Broca Area. NeuroImage, 13, part 2, 2001. ZIMMER, M. A leitura em língua estrangeira e os efeitos da freqüência e da consistência do insumo lexical em L2. Linguagem em (Dis)curso, Palhoça, SC, v.10, n.1, p. 111-131, 2010. 